\title{
Two-Case Series of Heart Transplant at a Single Center in a SARS-CoV-2 Epicenter
}

\author{
Marlena Sabatino ${ }^{1}$, Cassandra Soto ${ }^{1}$, Krish Dewan ${ }^{1}$, Joshua Chao $^{1}$, Hirohisa Ikegami ${ }^{2}$, \\ Anthony Lemaire ${ }^{3}$, Mark Russo ${ }^{1}$, and Leonard Lee ${ }^{4}$ \\ ${ }^{1}$ Rutgers Robert Wood Johnson Medical School \\ ${ }^{2}$ Robert Wood Johnson Foundation \\ ${ }^{3}$ Rutgers Robert Wood Johnson Medical School New Brunswick \\ ${ }^{4}$ Rutgers-Robert Wood Johnson Medical School
}

June 8, 2021

\begin{abstract}
As SARS-CoV-2 continues to challenge hospital systems, the safety of heart transplantation must be evaluated. Retrospective review of all heart recipients transplanted at a single academic medical center in a U.S. SARS-CoV-2 epicenter found two patients with non-ischemic dilated cardiomyopathy. The 34-year-old male (ejection fraction <10\%) was bridged to transplant with extracorporeal membrane oxygenation and microaxial left ventricular assist device. His perioperative course was uncomplicated except for transient SARS-CoV-2 seropositivity two months post-transplant. He was asymptomatic and remained so eight months to follow. The 20-year-old female (ejection fraction 5\%) was bridged to transplant with microaxial left ventricular assist device. She progresses well with SARS-CoV-2 seronegativity eight months post-transplant. Our early experience suggests that intentional recipient, donor, and provider testing, cautious organ procurement, strategic intrahospital patient organization and transport, and well-coordinated follow-up permits uninterrupted provision of this definitive therapy for heart failure without subjecting these patients to greater risk.
\end{abstract}

Title: Two-Case Series of Heart Transplant at a Single Center in a SARS-CoV-2 Epicenter

Running Head: Heart Transplant SARS-CoV-2 Epicenter

Authors \& Affiliations: Marlena E Sabatino BA ${ }^{1}$, Cassandra Soto $\mathrm{MD}^{1}$, Krish C Dewan MD ${ }^{1,2}$, Joshua C Chao MD, JD ${ }^{1,2}$, Hirohisa Ikegami $\mathrm{MD}^{1,2}$, Anthony Lemaire $\mathrm{MD}^{1,2}$, Mark J Russo MD, MS ${ }^{1,2}$, Leonard Y Lee $\mathrm{MD}^{1,2}$

${ }^{1}$ Rutgers Robert Wood Johnson Medical School, New Brunswick, NJ ${ }^{2}$ Robert Wood Johnson University Hospital, Department of Surgery, Division of Cardiothoracic Surgery, New Brunswick, NJ

\section{Email Addresses:}

Marlena E Sabatino, mes436@rwjms.rutgers.edu (ORCID ID : https://orcid.org/0000-0003-3029-3468)

Cassandra Soto, cs1162@rwjms.rutgers.edu

Krish C Dewan, krishdewan99@gmail.com

Joshua C Chao, joshua.c.chao@rutgers.edu

Hirohisa Ikegami, hirohisa.ikegami@rwjms.rutgers.edu

Anthony Lemaire, lemairan@rwjms.rutgers.edu 
Mark J Russo, mr1533@rwjms.rutgers.edu

Leonard Y Lee, leele@rwjms.rutgers.edu

Corresponding Author: Dr. Leonard Y Lee

Rutgers Robert Wood Johnson Medical School, 1 Robert Wood Johnson Place, East Tower, 8th Floor, Room 843, New Brunswick, NJ 08901 Tel: 732-235-9221, Fax: 732-448-0307

Data Availability: Data sharing not applicable to this article as no datasets were generated or analyzed during the current study.

Funding : None

Conflict of Interest: None

IRB Approval \& Patient Consent: The Rutgers Robert Wood Johnson Medical School Institutional Review Board has determined that this study meets the regulatory definition of non-human subject research; therefore, patient informed consent is waived.

Meeting Presentation: Eastern Cardiothoracic Surgical Society Annual Meeting; Virtual, 8 Oct 2020.

Word Count: 1479/1500

\section{Abstract}

As SARS-CoV-2 continues to challenge hospital systems, the safety of heart transplantation must be evaluated. Retrospective review of all heart recipients transplanted at a single academic medical center in a U.S. SARS-CoV-2 epicenter found two patients with non-ischemic dilated cardiomyopathy. The 34-yearold male (ejection fraction $<10 \%$ ) was bridged to transplant with extracorporeal membrane oxygenation and microaxial left ventricular assist device. His perioperative course was uncomplicated except for transient SARS-CoV-2 seropositivity two months post-transplant. He was asymptomatic and remained so eight months to follow. The 20-year-old female (ejection fraction 5\%) was bridged to transplant with microaxial left ventricular assist device. She progresses well with SARS-CoV-2 seronegativity eight months post-transplant. Our early experience suggests that intentional recipient, donor, and provider testing, cautious organ procurement, strategic intrahospital patient organization and transport, and well-coordinated follow-up permits uninterrupted provision of this definitive therapy for heart failure without subjecting these patients to greater risk.

Word Count: 148/150

\section{Introduction}

The SARS-CoV-2 pandemic has significantly impacted organ transplantation. Several concerns became abruptly ascendant: donor SARS-CoV-2 status, and the safety of traveling for organ procurement, inpatient admission for high-risk patients, and immunosuppression. Heart transplantation gained global interest, with SARS-COV-2 case-fatality rates characterized at $20-27 \%$ in past heart recipients. ${ }^{1,2}$ Investigation has been minimal in patients transplanted after SARS-CoV-2's emergence, though short-term outcomes have been largely benign. ${ }^{3-5}$

New Jersey rapidly became a SARS-COV-2 epicenter. State of emergency was declared on March 9, 2020. Elective surgery was suspended through May 26, 2020. During this period, two orthotopic heart transplantations were performed at our academic medical center. These are reported to enrich the discussion on providing definitive therapy to advanced stage heart failure patients during pandemic conditions. This study does not meet the regulatory definition of human subject research as determined by the Rutgers Robert Wood Johnson Medical School Institutional Review Board; therefore, the requirement for informed patient consent is waived.

\section{Case 1}


A 34-year-old African American man with nonischemic dilated cardiomyopathy (NDCM), heart failure with reduced ejection fraction (HFrEF) of $<10 \%$ (New York Heart Association (NYHA) Class IV, Stage D) status post implantable cardioverter defibrillator (ICD) placement, atrial fibrillation, and chronic kidney disease presented in acute decompensated heart failure and was listed UNOS status 6 for transplant. For three months after listing, he was managed outpatient with milrinone. He experienced multiple episodes of ventricular fibrillation requiring cardioversion, and eventually presented to the hospital severely hypervolemic, in cardiogenic shock. Venoarterial extracorporeal membrane oxygenation (ECMO) was placed concomitantly with an Impella 5.5 (Abiomed, Danvers, MA) device. Persistent right ventricular dysfunction prevented weaning form mechanical circulatory support. He was listed UNOS status 1.

Several weeks later, he underwent transplant. Preoperatively, he/donor tested SARS-CoV-2 negative. He experienced mild asymptomatic acute cardiac rejection (International Society for Heart and Lung Transplantation, Grade 1A) on myocardial biopsy, but no other complications. Standard immunosuppression was implemented after induction with mycophenolate mofetil. On postoperative day nine, he was discharged home. On postoperative day sixty, he tested SARS-CoV-2 positive upon routine screening before in-person follow-up but never exhibited symptoms. He continues to progress well at eight-month follow-up.

\section{Case 2}

A 20-year-old African American woman with NDCM, HFrEF of 20\%, and diabetes mellitus presented in acute decompensated heart failure thirteen months after ICD placement (EF 5\%, NYHA Class IV, Stage D). Impella 5.5 (Abiomed, Danvers, MA) was implanted. She was listed UNOS status 1. Ten weeks later, she underwent transplant after she/donor tested SARS-CoV-2 negative. Standard immunosuppression was implemented after induction with rabbit anti-thymocyte globulin and mycophenolate mofetil. On postoperative day ten, she was discharged home following an uncomplicated hospital course. She continues to progress well. Eight months postoperatively, she has consistently tested negative for SARS-CoV-2, with no evidence of rejection.

\section{Conclusion}

Situated in a SARS-COV-2 epicenter, our academic medical center designated all intensive care units as SARS-COV-2 units, except the cardiovascular unit (CVICU). Both patients were admitted to CVICU given their circulatory support requirements. No-visitor policies were implemented, and employees were instructed to self-monitor for SARS-COV-2 symptoms. A single provider was appointed as the contact for each patient to reduce total contacts, especially perioperatively when patients are maximally immunosuppressed.

New Jersey's Stay-At-Home order and recommendation against elective surgery likely aided in our center's ability to provide judicious emergent care, including care for patients in decompensated heart failure awaiting transplant. Under similar conditions, Hsu et al.in Los Angeles performed eight successful heart transplants at the University of California early in the pandemic. ${ }^{4}$

Regarding donor-to-recipient transmission risk, donor institutions confirmed SARS-CoV-2 negativity in both cases. Organ procurement was limited to single surgeon/restricted geographic radius. Besides utilizing more personal protective equipment intraoperatively, usual operative protocols were unchanged.

Postoperatively, both patients were transported to CVICU along designated SARS-CoV-2-negative pathways; Boffini et al. described comparable measures. ${ }^{6}$ We considered the risks of standard perioperative immunosuppression, i.e. , calcineurin and inosine-5'-monophosphate dehydrogenase inhibitors preventing $\mathrm{T}$ lymphocyte proliferation and thereby downregulating the body's defense against viruses like SARS-CoV-2. However, so too could immunosuppression diminish the susceptibility of heart recipients to inflammatory sequelae characteristic of SARS-CoV-2 infection. Thus, we elected to proceed with standard immunosuppression accompanied by serial myocardial biopsies to monitor for graft rejection. Patient education regarding self-quarantine and avoidance of infection risks supplemented usual institutional protocol. Consistent with our practice, current recommendations dictate continuation of standard immunosuppression with usual postoperative surveillance. ${ }^{6,7}$ No change was made to immunosuppression upon our Case 1 patient's seropositivity 
at two months post-transplant.

Concern of the morbidity of postoperative SARS-COV-2 has been a topic of consideration. An 87-patient study in China reported post-transplant patients experiencing virus-related morbidities comparable to those in the general population. ${ }^{4-6}$ In our study, one patient was transiently seropositive at two months posttransplant but was asymptomatic throughout the following eight months. This suggests that patients transplanted during the pandemic may be at no greater risk than the general population throughout their perioperative course. ${ }^{1,3,5}$ Even with these reassuring outcomes, minimal U.S. investigation begs further study.

At our academic medical center, interdisciplinary care coordination continues to minimize SARS-CoV-2 exposure risk. During a single visit, our heart recipients see their surgeon and cardiologist, and undergo myocardial biopsy. Telemedicine is also integrated into patient follow-up care, similar to Hsu et al .5

SARS-CoV-2 continues to challenge hospital systems globally. Reflecting on early pandemic experience, we demonstrate that intentional recipient, donor, and provider testing, cautious organ procurement, strategic intrahospital patient organization and transport, and well-coordinated follow-up may permit uninterrupted provision of definitive therapy for advanced stage heart failure without placing these patients at greater risk.

\section{References}

1. Iacovoni A, Boffini M, Pidello S, et al. A case series of novel coronavirus infection in heart transplantation from 2 centers in the pandemic area in the North of Italy. J Heart Lung Transplant. 2020;39(10):1081-1088.

2. Latif F, Farr MA, Clerkin KJ, et al. Characteristics and outcomes of recipients of heart transplant with coronavirus disease 2019. JAMA Cardiology. 2020;e202159.

3. Ren ZL, Hu R, Wang ZW, et al. Epidemiological and clinical characteristics of heart transplant recipients during the 2019 coronavirus outbreak in Wuhan, China: A descriptive survey report. J Heart Lung Transplant. 2020;39(5):412-417.

4. Hsu JJ, Al-Saffar F, Ardehali R, et al. Heart transplantation in the early phase of the COVID-19 pandemic: A single-center case series. Clinical Transplant. 2020;34(9):e14042.

5. Singhvi A, Barghash M, Lala A, et al. Challenges in heart transplantation during COVID-19: A single-center experience. J Heart Lung Transplant. 2020;39(9):894-903.

6. Boffini M, Pidello S, Simonato E, et al. An effective protocol for heart transplantation during COVID19 outbreak. Transplant International. 2020;33(10):1326-1328.

7. Aslam S, Danziger-Isakov L, Luong ML, et al. Guidance from the International Society of Heart and Lung Transplantation regarding the SARS CoV-2 pandemic. 2020;1-20. 